HU-EP-02/11

AEI-2002/026

hep-th/0204037

\title{
Perturbative Instabilities on the Non-Commutative Torus, Morita Duality and Twisted Boundary Conditions
}

\author{
Zachary Guralnik ${ }^{\dagger 1}$, Robert C. Helling ${ }^{\dagger 2}$, Karl Landsteiner ${ }^{\dagger 3}$ \\ and Esperanza Lopez $z^{\triangleleft}$ \\ $\dagger$ Institut für Physik \\ Humboldt-Universität zu Berlin, Invalidenstraße 110 \\ D-10115 Berlin, Germany \\ $\diamond$ Max Planck Institut für Gravitationsphysik \\ Albert Einstein Institut, Am Mühlenberg 1 \\ D-14476 Golm, Germany
}

\begin{abstract}
:
We study one-loop corrections in scalar and gauge field theories on the non-commutative torus. For rational $\theta$, Morita equivalence allows these theories to be reformulated in terms of ordinary theories on a commutative torus with twisted boundary conditions. UV/IR mixing does not lead to singularities, however there can be large corrections. In particular, gauge theories show tachyonic instabilities for some of the modes. We discuss their relevance to spontaneous $\mathbb{Z}_{N} \times \mathbb{Z}_{N}$ symmetry breaking in the Morita dual $S U(N)$ theory due to electric flux condensation.
\end{abstract}

\footnotetext{
1 zack@physik.hu-berlin.de

2 helling@AtDotDe.de

3 Karl.Landsteiner@physik.hu-berlin.de

4 lopez@aei-potsdam.mpg.de
} 


\section{Introduction}

For a variety of reasons, non-commutative field theories have been of much recent interest. One motivation for studying such theories is that the notion of space-time presumably has to be modified at very short distances $[\mathbf{S n}][\mathbf{C}][\mathbf{D F R}]$. Space-time non-commutativity also arises naturally in string theories with background fluxes (see [DN] and references therein). Interestingly, gauge theories on tori with magnetic flux and twisted Eguchi-Kawai models can be reformulated in terms of non-commutative gauge theories[GK][SZ].

In the simplest example of a non-commutative space, the space-time coordinates satisfy

$$
\left[x^{i}, x^{j}\right]=i \vartheta^{i j}
$$

with $\vartheta$ independent of $x$. One of the most striking differences with the commutative case is the phenomenon of ultraviolet-infrared mixing discovered in [MvRS]: in non-commutative spaces even massive theories can have amplitudes that are finite for generic external momenta but diverge as these external momenta are taken to zero, a divergence that one would usually classify as an IR phenomenon and that should be absent in a massive theory if the Wilsonian renormalization group picture holds.

UV/IR mixing has dramatic physical consequences. In the cases where UV/IR mixing leads to pole-like singularities there are two kinds of possible behaviors for the two-point function. The one-loop contributions can make the energies of low momentum modes grow. This happens for example in non-commutative $\phi^{4}$ theory. For these kind of theories it has been pointed out already in [MvRS] that a proper definition demands a resummation, not unsimilar to resummation techniques in finite temperature field theories. For non-commutative $\phi^{4}$ this program has been carried out explicitely in [GP] where renormalizability of this model has been proven.

A rather different situation arises in non-commutative pure Yang-Mills theories [Ha], [MST]. The one-loop correction contributes with a negative sign and leads to modes with imaginary energies at at low momentum [LLT1], [RR], [LLT2],[BGNV]. An important observation in this respect is that the non-planar one-loop graph can be interpreted as the two-point function of the open Wilson-line operators of non-commutative field theories [KRSY1], [KRSY2], [AL], [KKRS]. Recently it has been argued that the instabilities can be understood from a matrix model point of view as the non-cancellation of zero point energies giving rise to a potential between the "D0"-branes of a non-supersymmetric matrix model [vR].

In this paper we study similar phenomena on the non-commutative torus ${ }^{1}$. The UV-behavior of gauge theories on the non-commutative torus has been studied in [KW]

\footnotetext{
$1 \mathrm{UV} / \mathrm{IR}$ mixing on the fuzzy sphere has been studied in [CMS].
} 
and a discussion of a scalar $\phi^{3}$ model and gauge theory in the limit $\vartheta \rightarrow 0$ has appeared in [GMW]. In the compact case $\vartheta$ which has dimension length squared can be turned into a dimensionless parameter $\theta$ after dividing by the volume of the torus. For rational $\theta$, field theories on the non-commutative torus can be mapped by Morita equivalence to theories of matrix valued fields on an ordinary, commutative torus with twisted boundary conditions. For the detailed construction, see for example [GT] or [S]. For a discussion of rational/irrational $\theta$, see $[\mathbf{A B}][\mathbf{H}]$.

The field theory on this commutative space is equivalent to the non-commutative theory and yet should have properties that one would expect of local field theories. We will discuss why UV/IR mixing does not introduce new dependence on external momenta in the commutative theory with twisted boundary conditions after integrals over loop momenta in the non-compact theory have been replaced by discrete sums over modes appropriate for the torus. In this analysis we will find that even the commutative theory displays some unexpected behavior. For certain parameters, the renormalized dispersion relation will have tachyonic modes, as it was the case for the theory on the non-commutative plane.

The organization of the paper is as follows. In section two we discuss Morita duality and introduce conventions and notation. In section three we compute the one-loop corrections to the two-point functions in a scalar field model with Moyal-bracket interactions in dimensions $D=2,3,4$. The results are interpreted with respect to Morita duality and we also explicitely show how the two-point function develops discontinuities in $\theta$ at one-loop. In section four we discuss gauge theories. This turns out to be algebraically much more involved than the scalar field, however we are able to show that qualitatively the behavior of gauge theories is analogous to what happened in the scalar case. In section five we interpret the results of the previous section with respect to UV/IR mixing and Morita duality. In section six we analyze the consequences of the tachyonic mass terms we found for the phase structure of the gauge theory in $D=4$ with two non-compact dimensions. We argue that these instabilities arise from the spontaneous breaking of translation invariance in the non-commutative theory. In the Morita dual picture this corresponds to condensation of electric fluxes via spontaneous breaking of $\mathbb{Z}_{N} \times \mathbb{Z}_{N}$ symmetry. Some technical aspects of the calculations are collected in the appendices.

\section{The non-commutative torus and Morita equivalence}

In this chapter, we will introduce Morita equivalence and explain our conventions. We assume that two dimensions (denoted by $x^{1}$ and $x^{2}$ ) are compactified on a non-commutative

square torus of radius $R$, whereas the remaining $d=D-2$ are non-compact, commuting directions. For the non-commuting directions, we have

$$
\left[x^{i}, x^{j}\right]=i \vartheta^{i j}=2 \pi i R^{2} \theta \epsilon^{i j} .
$$


Note the difference between $\vartheta$ which is a dimensionful quantity and $\theta$ which is dimensionless. The distinction between rational and irrational makes only sense for a dimensionless quantity. We are primarily interested in the case of $\theta$ being rational. Furthermore, here $\epsilon^{12}=-\epsilon^{21}=1$. This commutation relation translates to a $*$-product

$$
(f * g)(x)=\left.\exp \left(\pi i R^{2} \theta \epsilon_{i j} \partial_{x}^{i} \partial_{y}^{j}\right) f(x) g(y)\right|_{y \rightarrow x}
$$

A field on the non-commutative torus can be expanded as

$$
\phi(\vec{x})=\sum_{\vec{k} \in \mathbb{Z}^{2}} \phi_{\vec{k}} e^{i \frac{\vec{x}}{R} \cdot \vec{k}}
$$

The ${ }^{*}$-product in terms of Fourier modes is then

$$
(\phi * \psi)(x)=\sum_{\vec{k} \in \mathbb{Z}^{2}}\left(\sum_{\vec{l} \in \mathbb{Z}^{2}} \phi_{\vec{l}} \psi_{\vec{l}-\vec{k}} e^{\pi i \theta \vec{k} \times \vec{l}}\right) e^{i \frac{\vec{x}}{R} \cdot \vec{k}}
$$

We can use the Baker-Campbell-Hausdorff formula $e^{A} e^{B}=e^{A+B} e^{\frac{1}{2}[A, B]}$ to split the exponent of non-commutative coordinates into two factors. For $\mathcal{U}=e^{i \frac{x_{1}}{R}}$ and $\mathcal{V}=e^{i \frac{x_{2}}{R}}$ we find the commutation relation

$$
\mathcal{U} * \mathcal{V}=\mathcal{V} * \mathcal{U} e^{-2 \pi i \theta}
$$

This implies

$$
\mathcal{U}^{k_{1}} * \mathcal{V}^{k_{2}} e^{\pi i \theta k_{1} k_{2}}=e^{i \frac{\vec{x}}{R} \cdot \vec{k}} .
$$

In the case of rational $\theta=p / N$ the commutation relation (2.1) can also be realized in terms of $N \times N$ clock and shift matrices

$$
U=\left(\begin{array}{cccc}
1 & & & \\
& e^{\frac{2 \pi i p}{N}} & & \\
& & \ddots & \\
& & & e^{\frac{2 \pi i p(N-1)}{N}}
\end{array}\right), \quad V=\left(\begin{array}{cccc}
0 & 1 & & \\
& 0 & 1 & \\
& & \ddots & \ddots \\
1 & & &
\end{array}\right)
$$

They obey the same algebra as the non-commutative Fourier modes $\mathcal{U}$ and $\mathcal{V}$. Thus, instead of a scalar field on the non-commutative torus we can equivalently study the matrix valued field

$$
\hat{\phi}(x)=\sum_{\vec{k} \in \mathbb{Z}^{2}} \phi_{\vec{k}} U^{k_{1}} V^{k_{2}} e^{\pi i \theta k_{1} k_{2}} e^{i \frac{\vec{x}}{R} \cdot \vec{k}}
$$

on a commutative torus (with ordinary commuting coordinates $\vec{x}$ ). 
The definition of the matrix generators (2.2) implies that $U^{N}=V^{N}=\mathbb{1}$. The matrices $U^{k_{1}} V^{k_{2}}$ for $k_{1}, k_{2}=0, \ldots, N-1$ are a basis of the $N \times N$ matrices. The mode indices $\vec{k} \in \mathbb{Z}^{2}$ thus label both matrix entries and momentum modes in (2.3). It is therefore useful to decouple these two meanings. One should decompose the labels as

$$
k_{i}=N r_{i}+\kappa_{i}
$$

with $\vec{r} \in \mathbb{Z}^{2}$ and $\vec{\kappa} \in \mathbb{Z}_{N}^{2}$. We will use Greek letters in the following for the "fractional" part of the momenta. In terms of these (2.3) becomes

$$
\hat{\phi}(x)=\sum_{r \in \mathbb{Z}^{2}} \sum_{\kappa \in \mathbb{Z}_{N}^{2}} \tilde{\phi}_{\vec{r}, \vec{\kappa}} U^{\kappa_{1}} V^{\kappa_{2}} e^{\pi i \theta \kappa_{1} \kappa_{2}} \exp \left(i \frac{\vec{x}}{R} \cdot \vec{\kappa}\right) \exp \left(\frac{i \vec{x} \cdot \vec{r}}{R / N}\right),
$$

where we changed the sign convention to $\tilde{\phi}_{\vec{r}, \vec{k}}=(-1)^{p\left(N r_{1} r_{2}+r_{1} \kappa_{2}+r_{2} \kappa_{1}\right)} \phi_{N \vec{r}+\vec{\kappa}}$. This should be read as follows: the last factor is the usual Fourier mode for fields on an $R / N \times R / N$ torus with discrete momentum $\vec{r}$. The sum over $\vec{\kappa}$ together with the matrix factor $U^{\kappa_{1}} V^{\kappa_{2}}$ is the basis decomposition of $N \times N$ matrices. Note especially that $\vec{\kappa}=(0,0)$ is the trace degree of freedom that we will also denote as the $U(1)$ part of the matrix valued field. We conclude that only the integer part $r_{i}$ of $k_{i} / N$ should really be thought of as momentum. We see that the commutative torus is smaller by a factor $N \times N$ than the non-commutative one. This is not surprising since upon this rescaling also the "density of degrees of freedom" is kept constant as now we are dealing with $N \times N$ matrices instead of scalars. The integral of the Lagrangian should be replaced by the integral of the trace of the new matrix valued Lagrangian.

However, $\hat{\phi}$ is not periodic when carried around the cycles of the smaller torus, rather there are holonomies due to the phase $\exp \left(i \frac{\vec{x}}{R} \cdot \vec{\kappa}\right)$ :

$$
\begin{aligned}
& \hat{\phi}\left(x_{1}+2 \pi R / N, x_{2}\right)=V^{m} \hat{\phi}(\vec{x}) V^{-m} \\
& \hat{\phi}\left(x_{1}, x_{1}+2 \pi R / N\right)=U^{-m} \hat{\phi}(\vec{x}) U^{m}
\end{aligned}
$$

where we defined $m=\frac{1}{N \theta}$. This of course should be interpreted properly in $\mathbb{Z}_{N}$. For $\theta=\frac{p}{N}$ this means $p m \equiv 1(\bmod N)$ or $p m-a N=1$ for some integer $a$ (this exists as $p$ and $N$ have no common divisors). It turns out[vB1][S] that in gauge theories modes with fractional momentum $\vec{\kappa}$ carry an electric flux

$$
e_{i} \equiv p \epsilon_{i j} \kappa_{j} \bmod N
$$

This relation can be inverted as $\kappa_{i} \equiv m \epsilon_{i j} e_{j} \bmod N$. 
This relation among field theories on different (non)-commutative spaces is an example of Morita equivalence. In general, Morita equivalence on the non-commutative torus relates a torus with $\theta$ to one with

$$
\theta^{\prime}=\frac{a \theta+b}{c \theta+d} \quad \text { for } \quad\left(\begin{array}{ll}
a & b \\
c & d
\end{array}\right) \in S L(2, \mathbb{Z}) .
$$

The radius, the size of the matrices and the twist transform as

$$
R^{\prime}=(c \theta+d) R \quad\left(\begin{array}{l}
N^{\prime} \\
m^{\prime}
\end{array}\right)=\left(\begin{array}{ll}
a & b \\
c & d
\end{array}\right)\left(\begin{array}{l}
N \\
m
\end{array}\right)
$$

Rational $\theta$ can be related to $\theta^{\prime}=0$, that is to a theory on a commutative space. This is the relation we deal with in this note.

For concreteness, we will first consider scalar field theories of the type

$$
\mathcal{L}=\frac{1}{2} \partial_{\mu} \bar{\phi} \partial^{\mu} \phi+\frac{1}{2} \mu^{2} \bar{\phi} \phi+a \phi * \phi * \bar{\phi} * \bar{\phi}+b \phi * \bar{\phi} * \phi * \bar{\phi}
$$

Our results do not rely on the fact that we are dealing with a scalar field, in fact they should be generally applicable for field theories on the non-commutative torus. Rather, we choose this example for notational simplicity. In a later chapter, we also discuss the case of pure gauge theory.

At the one-loop level, the $a$ and $b$ type interactions receive different contributions from planar and non-planar diagrams, as shown in Fig. 1. However, most of the time, we will specialize to the Moyal-bracket interaction

$$
[\phi, \bar{\phi}]_{*}^{2}
$$

since this resembles the form of the gauge theory interaction. This interaction term can be obtained from (2.8) by stetting $b=-a \equiv g^{2} / 2$. From Fig. 2 , we see, that for this choice the planar and non non-planar graphs contribute with weights 2 and -2 . This is especially convenient in the case of massless theories that might have (ordinary) IR divergencies: because of the relative minus sign between the planar and non-planar contribution these IR divergencies cancel and we can ignore them.

It is well known[GK][F] that the non-commutativity affects planar diagrams only through phase factors for the external legs. In contrast, non-planar diagrams contain phase factors that mix external momenta and loop momenta, thus the effect of non-commutativity is much more severe. In most of what follows we will just give the expressions for the nonplanar amplitudes. The corresponding planar amplitudes can then be read of by setting $\theta$ to zero in the Feynman integral. The total amplitude is just the difference for the 

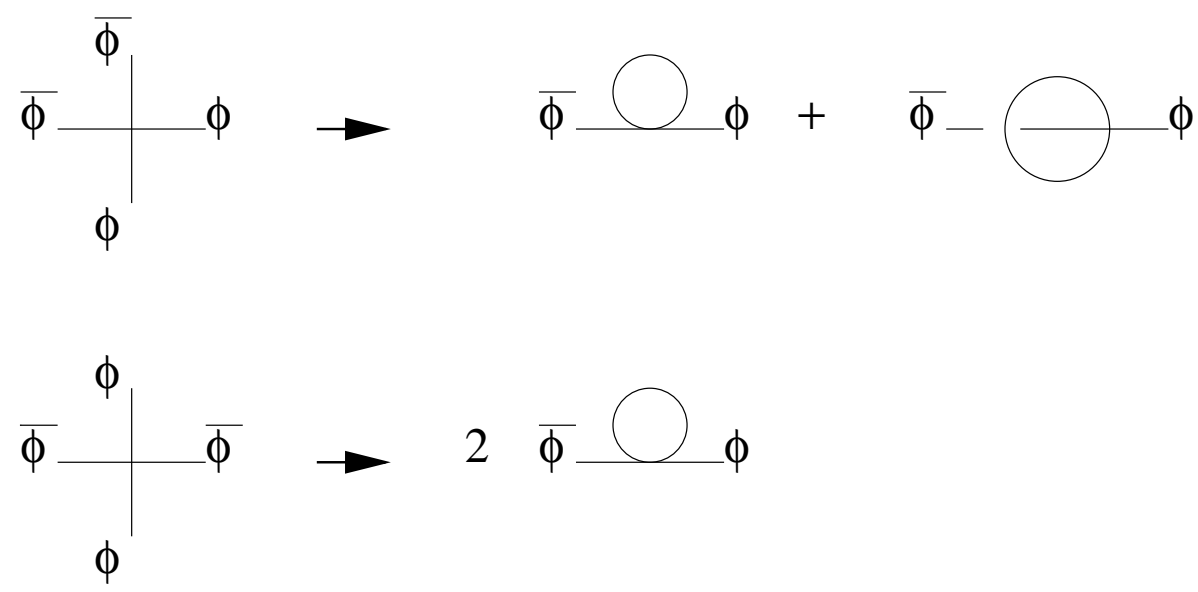

Fig. 1: The contributions of $\phi \phi \bar{\phi} \bar{\phi}$ and $\phi \bar{\phi} \phi \bar{\phi}$ at one-loop order

Moyal-bracket interaction. For more general choices of $a$ and $b$ in (2.8), one has to use the expressions shown in Fig. 1 to derive the correct relative factors.

In [MvRS], non-planar one loop diagrams were studied. The simplest example being

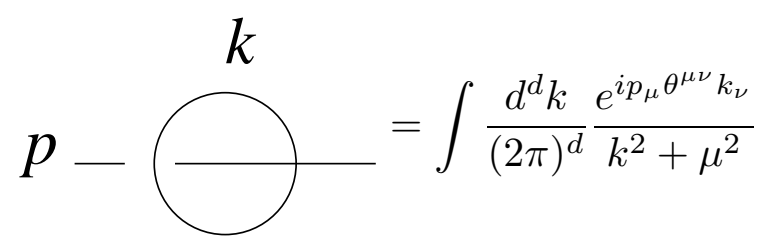

The exact value for this integral will of course depend on the dimension $d$, but it is easy to qualitatively anticipate the result: without non-commutativity, the mass makes the integral well behaved for small $k$. For large $k$ the integral will diverge logarithmically for $d=2$ and like $k^{d-2}$ for higher dimensions.

In the presence of the phase factor, the integrand oscillates and in fact the integral converges for large $k$. The combination $p \circ p:=p_{\mu} \theta^{\mu \nu} \theta_{\nu \rho} p^{\rho}$ has dimension length squared. As a dimensionful quantity, it can serve as a regulator. In fact, at leading order, the two dimensional integral behaves like $\log (p \circ p)$ and in higher dimensions like

$$
\frac{1}{(p \circ p)^{\frac{d-2}{2}} .}
$$

We see, that the non-commutativity introduces a non-trivial dependence on the external momentum. Of course, if there is no external momentum in the non-commutative directions, that is $\theta^{\mu \nu} p_{\nu}=0$, this regulator vanishes and the integral again diverges.

This non-trivial dependence and especially the divergence for $p \rightarrow 0$ for massive theories came as a surprise in [MvRS] and has been termed "UV/IR-mixing". The ultraviolet divergencies reentering for infrared values of external momenta even casts doubt on the validity of the Wilsonian renormalization group in non-commutative theories. 
On the other hand, as we have explained above, on the torus with rational values of $\theta$, the non-commutative theory can be reformulated in terms of a commutative theory with matrix valued fields and twisted boundary conditions. This commutative theory is not expected to contain any such surprises and thus should not show any signs of UV/IRmixing. It is one of the topics of this paper to resolve this puzzle.

\section{One loop on the torus}

Now, that we have set the scene, we can perform the actual calculation in the scalar field model in $2+d$ Euclidean dimensions. The interesting non-planar amplitude (2.9) takes then the form

$$
\begin{aligned}
A(\vec{n}) & =\int \frac{d^{d} k}{(2 \pi)^{d}} \frac{1}{(2 \pi R)^{2}} \sum_{\vec{l} \in \mathbb{Z}^{2}} \frac{e^{i 2 \pi \theta l_{j} n_{k} \epsilon_{j k}}}{k^{2}+\frac{\vec{l}^{2}}{R^{2}}+\mu^{2}} \\
& =\int \frac{d^{d} k}{(2 \pi)^{d}} \frac{1}{(2 \pi R)^{2}} \sum_{\vec{l} \in \mathbb{Z}^{2}} \int_{0}^{\infty} d \alpha e^{-\alpha\left(k^{2}+\frac{\vec{l}^{2}}{R^{2}}+\mu^{2}\right)+i 2 \pi \theta l_{j} n_{k} \epsilon_{j k}}
\end{aligned}
$$

The inflowing momentum is $\vec{n} / R$. Note that the amplitude does not depend on the external momentum in the commutative directions. Here, we have introduced a Schwinger parameter $\alpha$. In the following we will use $b_{j}=\theta \epsilon_{j k} n_{k}$

Now we can integrate out the momenta corresponding to the commuting directions. After a Poisson resummation the amplitude can be written as

$$
A=\frac{\pi^{d / 2+1}}{(2 \pi)^{d+2}} \int_{0}^{\infty} d \alpha \alpha^{-1-\frac{d}{2}} \sum_{\vec{l} \in \mathbb{Z}^{2}} e^{-\frac{(\vec{l}+\vec{b})^{2} R^{2} \pi^{2}}{\alpha}-\alpha \mu^{2}}
$$

Let us first treat the massless case. We finally integrate over the Schwinger parameter and arrive at

$$
A=\frac{\pi^{1-\frac{d}{2}}}{(2 \pi R)^{d}} \frac{\Gamma\left(\frac{d}{2}\right)}{(2 \pi)^{2}} \zeta(d / 2, \vec{b}) .
$$

In the last line we introduced the Epstein zeta function

$$
\zeta(s, \vec{b})=\sum_{l \in \mathbb{Z}^{2}}^{\prime} \frac{1}{\left[(\vec{l}+\vec{b})^{2}\right]^{s}} .
$$

We see that the only effect of the non-commutativity is to shift the momentum lattice for the non-planar graph. All oscillating phase factors have been removed by the resummation.

Of course this representation of the zeta-function as an infinite sum is only convergent for $\operatorname{Re}(s)>1$. Moreover, the sum is defined in such a way as to exclude lattice points where the denominator under the sum could vanish, i.e. for $\vec{b}=0$ we exclude the origin 
$\vec{l}=(0,0)$. This is denoted by the symbol ' in the sum. It turns out that for $d>0$ this is nothing but a UV-regularization since after the Poisson resummation the UV is $\vec{l}=(0,0)$. We see that this UV-divergence is regulated by the non-commutativity. The situation at $d=0$ is slightly different as we will explain in the next paragraph. We also consider the vector $\vec{b}$ to be reduced on the lattice, $b_{j} \in\left(-\frac{1}{2}, \frac{1}{2}\right]$. This reduction on the lattice is evident from the definition of the amplitude in formula (3.1) since the integer part of the vector $\vec{b}$ does not contribute to the expression. Alternatively, one can apply a shift to the sum over the dual lattice in (3.4).

As is well-known, the Epstein zeta function can be analytically continued over the complex $s$-plane with the help of Jacobi's theta-function in the form

$$
\begin{aligned}
\zeta(s, \vec{b})= & \frac{\pi^{s}}{\Gamma(s)}\left\{\frac{1}{s-1}-\frac{\delta_{\vec{b}, 0}}{s}+\right. \\
& \left.+\int_{1}^{\infty} d t\left[t^{-s}\left(\prod_{j=1}^{2} \vartheta\left(i t, b_{j}\right)-1\right)+\left(t^{s-1} e^{-\pi t \vec{b}^{2}} \prod_{j=1}^{2} \vartheta\left(i t, i t b_{j}\right)-\delta_{\vec{b}, 0}\right)\right]\right\}
\end{aligned}
$$

This representation is explained in appendix A.

Taking together planar and non-planar graphs in the theory with Moyal bracket interactions, the one-loop effective action can now be written as

$$
\Gamma_{2}^{(1)}=k^{2}+\frac{\vec{n}^{2}}{R^{2}}+\frac{g^{2} R^{2-d} \Gamma\left(\frac{d}{2}\right)}{2^{d} \pi^{\frac{3 d+2}{2}} R^{2}}(\zeta(d / 2, \overrightarrow{0})-\zeta(d / 2, \vec{b})) .
$$

Here $k^{2}$ denotes the momentum in the uncompactified dimensions (if existent). If there is a non-compact direction we can undo the Wick rotation and interpret the expression $\Gamma_{2}^{(1)}=0$ as a dispersion relation. $g^{2} R^{2-d}$ is a dimensionless parameter that should be small in order for perturbation theory to hold.

Whenever $\vec{b}=0$ planar and non-planar contribution cancel each other exactly. This can be easily understood in terms of the Morita-dual theory. Recalling the definition of $\vec{b}$ one sees that $\vec{b}=0$ corresponds to momentum modes $\vec{n}=N \vec{r}$. Morita duality maps these modes into the momentum $\vec{r}$ modes of the overall $U(1)$ degree of freedom. The $U(1)$ modes are non-interacting for Moyal bracket interaction and therefore the one-loop correction vanishes.

The modes for which $\vec{b} \neq 0$ are mapped to the $S U(N)$ degree of freedom under Morita duality. Since the one-loop correction depends only on the parameter $\vec{b}$ the amplitude is thus periodic under $\vec{n} \rightarrow \vec{n}+N \vec{r}$, with $\vec{r} \in \mathbb{Z}^{2}$. Therefore, in terms of the Morita dual theory these corrections are mass terms.

Let us discuss now the poles in in the zeta function at $s=0$ and $s=1$. In $D=2$, that is $s=0$ the zeta function vanishes except at the point $\vec{b}=0$. However, the gamma function 
in the denominator in (3.5) is canceled by the Gamma function in the Amplitude in (3.3). Therefore the amplitude has a pole for $\vec{b}=0$. The nature of this divergence is easily understood by examining (3.1). For $D=2$ this expression has a divergence arising from the zero-mode on the torus $(\vec{n}=0)$. The sum in (3.1) should then be defined by omitting the zero mode. It is this divergence that is regulated by the zeta-function expression for the amplitude. At $D=2$ the zeta function regulates the IR but leaves us with the UV divergence. We can now regulate the UV by subtracting the pole at $s=0$ in the brackets in (3.5) for the definition of the amplitude. ${ }^{2}$

There is no pole for $D=3$. The situation with two commuting, compact dimensions $(D=4)$ presents a new feature. There is a pole in (3.5) at $s=1$. This can be understood as follows. At low energies the theory reduces effectively to a two-dimensional field theory. Thus this field theory will have an infrared divergence as is common in two dimensions. We will not further worry about this infrared divergence since the form of the Moyalbracket interaction provides automatically an IR-regularization. Indeed the poles at $s=1$ cancel between the planar and non-planar contributions. Basically this is the statement that the theory is non-interacting at distances large compared to the radius of the torus. There is however a subtlety here. Due to one-loop effects the modes that are important at low energies are not only the zero modes on the torus. Again the contribution of the non-planar diagram can lower the energy of a momentum mode on the torus significantly. So there might be finitely many other modes that are important in the infrared as we will now discuss.

It can happen that for low momentum modes and large $N$ (large gauge group in the Morita dual theory) the contribution of the non-planar modes does not only dominate the planar one but also becomes larger than the tree-level term. If $\theta \epsilon_{j k} n_{k}$ is very close to a lattice point $\vec{b}$ becomes very small and for $\vec{b} \rightarrow \overrightarrow{0}$ the $\zeta$ function grows without bound. Expanding the effective action for small $\vec{b}$ we find

$$
\begin{aligned}
& \Gamma_{2} \approx \frac{\vec{m}^{2}}{R^{2}}+\frac{2 g^{2}}{\pi} \log (|\vec{b}|), \quad D=2 \\
& \omega^{2} \approx \frac{\vec{m}^{2}}{R^{2}}-\frac{g^{2}}{2 \pi^{2} R} \frac{1}{|\vec{b}|}, \quad D=3 \\
& \omega^{2} \approx k^{2}+\frac{\vec{m}^{2}}{R^{2}}-\frac{g^{2}}{4 \pi^{4} R^{2}} \frac{1}{\vec{b}^{2}}, \quad D=4 .
\end{aligned}
$$

Since the contribution of the non-planar diagram has negative sign this means that mass squared becomes negative.

${ }^{2}$ Of course, this leaves us with a finite undetermined constant. This is nothing but the usual mass renormalization. Notice that this is a universal mass for all $S U(N)$ modes in contrast to the corrections arising from the non-planar amplitude. 
It is convenient to label the modes by $\vec{e}$ according to (2.7) even if the interpretation as "electric" flux only makes sense in the gauge theory. We see then that $\vec{b}=\frac{1}{N} \vec{e}$. Therefore the non-planar contribution becomes maximal for unit electric flux in the Morita dual language. This does not necessarily mean that the unit electric fluxes are also the ones that become unstable. It might happen that $e_{i}=1$ corresponds to a rather large $n_{i}$. The tree level term might then still be larger than the one-loop correction resulting in a positive inverse two-point function for this mode. In this case unstable modes can first appear for larger electric fluxes.

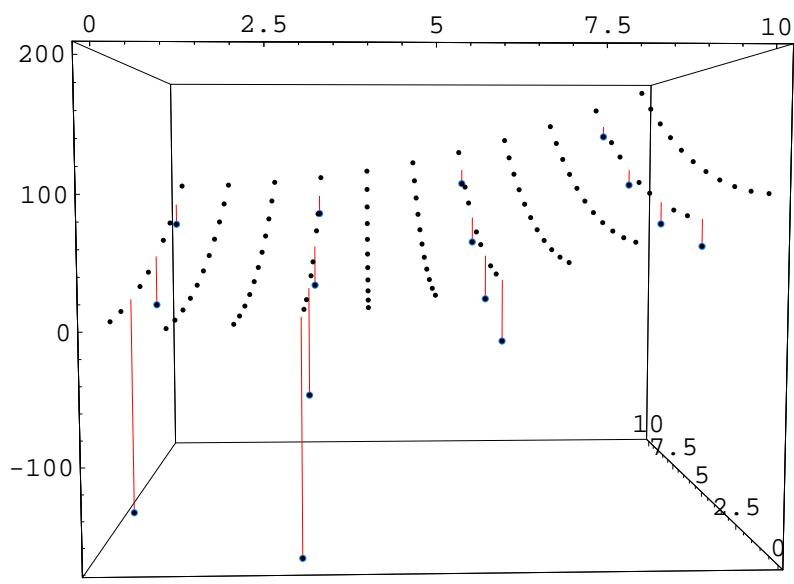

Fig. 2: This graph shows the dispersion relation for $\theta=\frac{1000}{3001}$.

To illustrate this point let us work out a concrete example in $D=4$. The graph in Fig. 2 shows a typical plot of the dispersion relation. We chose $\theta=1000 / 3001$ such that $\vec{b}$ becomes very small for every third mode in the momentum lattice on the non-commutative torus. For low lying modes the one-loop correction dominates the tree level term and the mode becomes unstable. The (non-commutative) momentum modes corresponding to unit electric flux are in this case $n_{i}=3$. If we keep the radius $R$ and the coupling constant $g$ fixed and take $\theta=1000 / 3003$ instead the unit electric flux is mapped to the momentum mode $n_{i}=1000$. Although this is the mode for which the one-loop correction is maximal it is not necessarily tachyonic since the tree-level term is much larger. The most tachyonic modes will still be the momentum modes $(3,0)$ and $(0,3)$ as before (corresponding to the electric flux $e=3$ ). Of course had we chosen $\theta=1 / 3$ instead, the corrections for every third mode would vanish identically.

Let us compare our findings with the behavior in the non-compact case where it is well known that low momentum modes can become tachyonic at one-loop[LLT2][RR][BGNV]. We could have expected that at least for very large radius the theory on the torus should behave similar as the non-compact one. Of course in the non-compact case there is no mass-gap and the one-loop contribution grows arbitrarily large for low momentum. On the torus the 
zero mode behaves completely benign. Nevertheless low, but non-zero, momentum modes can become unstable. For large momenta the tree level term grows quadratically and will certainly dominate over the one-loop correction. What is perhaps somewhat surprising here is the fact that tachyonic behavior appears even in the case with rational $\theta$. One might have expected that this peculiar feature of non-commutative theories is absent here since there is a Morita dual description in terms of a conventional $U(N)$ theory on a commutative torus, where one does not naively expect any UV/IR mixing. We emphasize that instabilities appear only for large (but finite) $N$.

In the case of irrational $\theta$, integer multiples of $\theta$ get arbitrarily close to integers. The question of course remains whether the one-loop correction can compete with the tree level term that is quadratic in the external momenta. The "pigeon hole theorem" of rational approximation theory implies that there are infinitely many integers $n$ such that $n \theta$ is closer to an integer than $1 / n^{2}$. That is, in $D>4$ there are always infinitely many unstable modes. For $D=4$, the two terms are generically of the same order and the existence of unstable modes irrespective of $\theta$ depends on the coupling $g$. For $D<4$ and for generic $\theta$ there need not be unstable modes. However, there are irrational numbers $\theta$ such that the estimate $1 / n^{2}$ above can improved to any power $1 / n^{a}$ and hence for those values of $\theta$ there are infinitely many unstable modes in all dimensions.

Let us now have a look at the massive case. Integrating the amplitude (3.2) over $\alpha$ gives

$$
A=\frac{(R \mu)^{\frac{d}{2}}}{(2 \pi)(2 \pi R)^{d}} \sum_{\vec{l} \in \mathbb{Z}_{2}}{ }^{\prime} \frac{1}{\left[(\vec{l}+\vec{b})^{2}\right]^{\frac{d}{4}}} K_{\frac{d}{2}}\left(2 \pi \mu R \sqrt{(\vec{l}+\vec{b})^{2}}\right) .
$$

$K_{\frac{d}{2}}$ denotes a modified Bessel-function. Since the Bessel-function decays exponentially for large $\vec{l}$, there is no $\frac{1}{s-1}$ pole connected to an IR singularity. The behavior at small $\vec{b}$ is the same as in the massless case. In each of the cases for small enough $|\vec{b}|$ the one-loop correction dominates the tree-level term. Independently of the presence of a mass-term we find therefore tachyonic instabilities.

We have limited ourselves to the one-loop approximation. One must ask the question whether this is reliable. Notice that $\vec{b}$ acted as a UV-regulator. In the case $D=4$ we are dealing with a renormalizable field theory. Therefore $n$-loop corrections to the two point function will at most behave quadratically in the cutoff and therefore contribute terms of the order $\frac{g^{2 n}}{\vec{b}^{2}}$. This is clearly sub-leading at small coupling. For $D<4$ the models are super-renormalizable and therefore higher loop terms will scale with lower powers of the cutoff than the one-loop term. In the non-compact case there is however an additional source for divergencies. It stems from the fact that insertions of the nonplanar one-loop graph into higher-order loop graphs can lead to new divergencies due to the infrared singular behavior of the one-loop correction. From our results it is evident 
that no such difficulty arises in the compact case with rational $\theta$. Indeed the non-planar one-loop contribution is perfectly non-singular on the complete lattice of non-commutative momenta. However, there could still appear terms of order $\frac{g^{2 n}}{\vec{b}^{2 n}}$ in these graphs. As argued in [MvRS] such difficulties can be handled by resumming the propagator. In the concrete model we considered here the resummed propagator leads to tachyonic pole. In this case one has to find first the effective potential and its minimum before proceeding in the loop expansion. In any case the tachyonic behavior can not be ignored.

\section{Gauge Theory on the NC Torus}

In this section we will consider a $U(1)$ gauge theory on the non-commutative torus. If the non-commutative parameter $\theta$ is rational, Morita duality relates this theory to an ordinary $U(N)$ theory on a torus with magnetic flux $m$. It is thus of special interest to study this case.

In order to analyze the quantum corrected dispersion relations we need to evaluate the polarization tensor. Its non-planar part is given by

$$
\Pi_{\mu \nu}^{N P}=\frac{g^{2}}{(2 \pi R)^{2}} \sum_{\vec{l} \in \mathbb{Z}^{2}} \int \frac{d^{d} k}{(2 \pi)^{d}} \frac{\cos \left(2 \pi \theta l_{i} n_{j} \epsilon_{i j}\right)}{K^{2}(K-P)^{2}} f_{\mu \nu}\left(P_{\rho}, K_{\sigma}\right)
$$

where as before $d$ is the number of commutative non-compact directions. We denote $\mu=(\alpha, i)$, with $\alpha=1, . ., d$ labeling the non-compact dimensions and $i=1,2$ the two directions of the non-commutative torus. The inflowing momentum is $P_{\mu}=\left(p_{\alpha}, n_{i} / R\right)$ and the loop momentum $K_{\mu}=\left(k_{\alpha}, l_{i} / R\right)$. In this section capital letters denote $D$ dimensional momenta. From now on we will set $R=1$ in order to simplify our expressions; it can be reintroduced by dimensional analysis. The polynomial $f_{\mu \nu}$ is [PS]

$f_{\mu \nu}=4 d K_{\mu} K_{\nu}-2 d\left(P_{\mu} K_{\nu}+P_{\nu} K_{\mu}\right)+(d-4) P_{\mu} P_{\nu}-g_{\mu \nu}\left(2 d K^{2}-(4 d+2) K \cdot P+(2 d-3) P^{2}\right)$.

Integrating the loop momentum along the non-compact directions, Poisson resumming and using standard properties of Bessel functions, we can rewrite (4.1) as

$$
\Pi_{\mu \nu}^{N P}=\frac{g^{2}}{(4 \pi)^{\frac{d}{2}+1}} \sum_{\vec{l} \in \mathbb{Z}^{2}} \int_{0}^{1} d x \cos (2 \pi P \cdot L x) \int_{0}^{\infty} \frac{d \alpha}{\alpha^{\frac{d}{2}}} e^{-\alpha x(1-x) P^{2}-\frac{\pi^{2}}{\alpha} L^{2}} h_{\mu \nu}\left(P_{\rho}, L_{\sigma}\right)
$$

where we have introduced $L_{\mu}=\left(0, l_{i}-\theta \epsilon_{i j} n_{j}\right)$ and

$$
\begin{aligned}
h_{\mu \nu}\left(P_{\rho}, L_{\sigma}\right)= & -\frac{4 d \pi^{2}}{\alpha^{2}}\left[L_{\mu} L_{\nu}-\left(P_{\mu} L_{\nu}+P_{\nu} L_{\mu}-g_{\mu \nu} P \cdot L\right) \frac{P \cdot L}{P^{2}}\right]- \\
& -\left(P_{\mu} P_{\nu}-g_{\mu \nu} P^{2}\right)(4 d x(1-x)-d+4) .
\end{aligned}
$$


We refer to Appendix B for a detailed derivation of these expressions. The planar part of the polarization tensor can be obtained from (4.2)-(4.3) by setting $\theta=0$. Notice that both terms in the rhs of (4.3) are manifestly transverse, so that $P^{\mu} h_{\mu \nu}=0$. The second term is proportional to the tree level two-point function. It contributes to the effective coupling constant and does not modify the form of the dispersion relation at 1-loop order. We will concentrate thus in the first term. Notice that this term is proportional to the number of degrees of freedom of the gauge field, i.e. $d=D-2$. Thus the cases of interest in this section will be $d=1,2$.

The $\alpha$ integration in (4.2) can be easily performed

$$
\begin{aligned}
& \int_{0}^{\infty} \frac{d \alpha}{\alpha^{\frac{d}{2}+2}} e^{-\alpha P^{2} x(1-x)-\frac{\pi^{2}}{\alpha} L^{2}}= \\
& \quad=2\left(\frac{\sqrt{P^{2} x(1-x)}}{\pi|L|}\right)^{\frac{d}{2}+1} K_{\frac{d}{2}+1}\left(2 \pi|L| \sqrt{P^{2} x(1-x)}\right) .
\end{aligned}
$$

We will approximate the sum over $\vec{l} \in \mathbb{Z}^{2}$ by keeping only the vector $\vec{l}_{0}$ at which $|L|$ acquires its minimum. This approximation is best for external momentum $\vec{n}$ such that $|L| \sim 1 / N$. In analogy with the previous section we denote the value of $L$ at $\vec{l}_{0}$ by $B_{\mu}=\left(0, b_{i}\right)$, where $b_{i}=\left(l_{0}\right)_{i}-\theta \epsilon_{i j} n_{j}$. These are the momenta for which we expect large 1-loop corrections. Furthermore we assume that $|B||P|$ is small. It turns out that the solution to the 1-loop corrected dispersion relation satisfies this requirement. The expansion of the modified Bessel function (4.4) at small values of the argument is

$$
K_{\frac{d}{2}+1}(2 z)=\frac{\Gamma\left(\frac{d}{2}+1\right)}{2 z^{\frac{d}{2}+1}}-\frac{\Gamma\left(\frac{d}{2}\right)}{2 z^{\frac{d}{2}-1}}+\ldots .
$$

Given that (4.3) is transversal, each contribution to the polarization tensor derived from expanding $K_{\frac{d}{2}+1}$ will satisfy independently the Ward identity.

Denote the contributions of the polarization tensor associated with the two terms in (4.5) by $\Pi^{N P, 1}$ and $\Pi^{N P, 2}$. Substituting the expansion into (4.2), we obtain

$$
\Pi_{i j}^{N P, 1}=\frac{d g^{2} \Gamma\left(\frac{d}{2}+1\right)}{2^{d} \pi^{\frac{3}{2} d+1}} \frac{b_{i} b_{j}}{|\vec{b}|^{d+2}} \delta_{0, \vec{n} \cdot \vec{b}}
$$

with all other components vanishing. The delta function arises from the $x$-integration of $\cos (2 \pi P \cdot B x)$, since $P \cdot B=\vec{n} \cdot \vec{b}$. The non-compact limit is achieved by setting $\theta=1 / N$ with $N \rightarrow \infty$. In this limit it is immediate to see that (4.6) reproduces the polarization tensor on $\mathbb{R}^{d} \times \mathbb{R}_{\theta}^{2}[\mathbf{H a}],[\mathrm{MST}]$.

From the next to leading term in (4.5) we obtain

$$
\Pi_{\mu \nu}^{N P, 2}=\frac{d g^{2} \Gamma\left(\frac{d}{2}\right)}{2^{d} \pi^{\frac{3}{2} d-1}} \frac{c(\vec{n} \cdot \vec{b})}{|\vec{b}|^{d}}\left[B_{\mu} B_{\nu} P^{2}-\left(P_{\mu} B_{\nu}+P_{\nu} B_{\mu}-g_{\mu \nu} P \cdot B\right) P \cdot B\right],
$$


where

$$
c(\vec{n} \cdot \vec{b})=\int_{0}^{1} d x x(1-x) \cos (2 \pi x \vec{n} \cdot \vec{b})=\left\{\begin{array}{cl}
\frac{1}{6} & \vec{n} \cdot \vec{b}=0 \\
-\frac{1}{2 \pi^{2}(\vec{n} \cdot \vec{b})^{2}} & \vec{n} \cdot \vec{b} \neq 0
\end{array}\right.
$$

The contribution at $\vec{n} \cdot \vec{b}=0$ has the same structure as (4.6) but is clearly sub-leading with respect to it. For $\vec{n} \cdot \vec{b} \neq 0$ (4.7) shows a more involved structure, whose origin is the requirement of preserving transversality. These modes also suffer corrections of order $g^{2} /|\vec{b}|^{d}$. Thus we see that regardless whether $\vec{n} \cdot \vec{b}$ vanishes or not there are always corrections of this order. Further terms in the expansion of the Bessel function at small argument are sub-leading.

We will study now the dispersion relations. The quantum corrected inverse propagator is given by

$$
\Gamma_{\mu \nu}^{(1)}=\left(\omega^{2}-\vec{n}^{2}\right) g_{\mu \nu}-\Pi_{\mu \nu}^{N P, 1}-\Pi_{\mu \nu}^{N P, 2} .
$$

We have assumed that the momentum along the spatial non-compact direction vanishes. Finding the poles of the propagator is involved because of the complicated, non-diagonal structure of $\Pi^{N P, 2}$. We will not attempt to solve the problem in the general case. Our aim is to analyze the possible appearance of unstable modes. This can only happen if the quantum corrections dominate over the tree level term. We will see that this condition is never met for modes with $\vec{n} \cdot \vec{b} \neq 0$. When $\vec{n} \cdot \vec{b} \neq 0$ the following relations hold

$$
|\vec{b}|=\left|\overrightarrow{l_{0}}-\theta \epsilon \cdot \vec{n}\right|>\frac{\left|\vec{l}_{0} \cdot \vec{n}\right|}{|\vec{n}|} \geq \frac{1}{|\vec{n}|}
$$

The dominance of the 1-loop correction in (4.8) requires $g^{2} /|\vec{b}|^{d} \geq \vec{n}^{2}$. For $d=1,2$ and small coupling, this is not possible for modes satisfying (4.9). Thus we will concentrate in momentum modes with $\vec{n} \cdot \vec{b}=0$. In this case, $\Pi^{N P, 2}$ is negligible with respect to $\Pi^{N P, 1}$. The dispersion relation of the tranverse photon polarized along the non-commutative torus for these modes reduces to

$$
\omega^{2}=\frac{\vec{n}^{2}}{R^{2}}-\frac{d g^{2} \Gamma\left(\frac{d}{2}+1\right)}{(2 R)^{d} \pi^{\frac{3}{2} d+1}} \frac{1}{|\vec{b}|^{d}},
$$

where we have reintroduced the dependence on the radius of the torus. This relation is analogous to the one obtained for the scalar model and thus the analysis performed there applies. For sufficiently large $N$ there are momentum modes that acquire negative energy square, giving rise to perturbative instabilities. Among the modes that satisfy $\vec{n} \cdot \vec{b}=0$ are those with momentum proportional to $(1,0)$ or $(0,1)$. It is easy to see that they are actually the first candidates to become perturbatively unstable. The analysis of the instability and its interpretation in terms of the Morita dual theory are left for section 6 . 


\section{UV/IR mixing on the torus?}

One of the major surprises of field theories on the non-commutative plane is the phenomenon of UV/IR mixing[MvRS]. Loop amplitudes in these theories show an unusual dependence on momenta. Even massive theories that are normally well behaved in the infrared have new divergences as $p \rightarrow 0$.

Here we will discuss UV/IR mixing in view of our results on the non-commutative torus for rational $\theta$. In this case there is a Morita equivalent commutative theory in terms of matrix valued fields with twisted boundary conditions. As the two theories are equivalent, one would expect some manifestation of UV/IR mixing in terms of the commutative theory. We are going to argue that in fact on the torus the usual low energy analysis holds. Recall the splitting of the non-commutative momentum as in (2.4). The amplitudes in the commutative theory with twisted boundary conditions do not show any unusual momentum dependence. Especially there are no new divergencies in the low energy regime. What appears as an unusual momentum dependence of loop amplitudes in the non-commutative picture becomes a certain dependence on the electric fluxes, or equivalently Lie algebra degrees of freedom, in the commutative description.

The main difference between the non-compact and compact case is that momentum integrations are replaced by discrete sums over the momentum modes. This allowed us to apply the Poisson resummation to translate the oscillatory factor to a shift in sum over modes. For concreteness let us discuss the case with two compact and two non-compact directions. There we derived for the one loop self-energy correction the expression (3.3). The external momentum entered as

$$
\sum_{\vec{k} \in \mathbb{Z}^{2}}{ }^{\prime} \frac{1}{(\vec{k}-\theta \vec{p})^{2}}
$$

If we rewrite this for rational $\theta=p / N$ using the splitting (2.4), we see that possibly after a relabeling the sum only depends on the "matrix entry" part $\pi_{i}$ of the momentum $\vec{p}=N \vec{r}+\vec{\pi}:$

$$
\sum_{\vec{k} \in \mathbb{Z}^{2}}{ }^{\prime} \frac{1}{\left(\vec{k}-\frac{p}{N} \vec{\pi}\right)^{2}}
$$

This rewriting demonstrates explictly that there is no unusual dependence on the commutative torus momenta $\vec{r}$. Thus this amplitude does not show UV/IR-mixing.

Of course not all amplitudes depend only on the fractional momenta. This is only the case if the corresponding planar amplitude is independent of the external momenta. Generically, dependence on momenta and electric fluxes mixes. This happens for example in the gauge theory two point function or for the one loop correction to the four point function (the fish-graph). Let us consider this case in a bit more detail. 


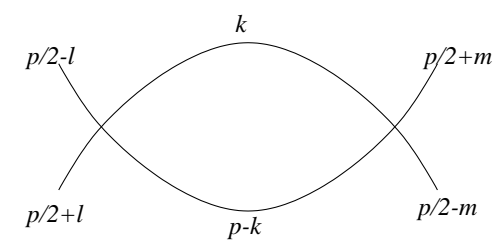

Fig. 3: The fish graph

The amplitude after Poisson resummation is proportional to

$$
\sum_{\vec{k} \in \mathbb{Z}^{2}} \int_{0}^{1} d x \int_{0}^{\infty} d \alpha e^{-\frac{\pi^{2}}{\alpha}(\vec{k}-\theta \vec{p})^{2}+2 \pi i(1-x) \vec{p} \cdot(\vec{k}-\theta \vec{p})+\alpha\left(x^{2}-x\right) \vec{p}^{2}}
$$

The external momentum $\vec{p}$ appears in two different ways: by itself and in the combination $\vec{k}-\theta \vec{p}$. The latter reduces to $\vec{k}$ in the commutative case $\theta=0$ so the former is the usual momentum dependence of this amplitude in a commutative theory. The only effect of the non-commutativity is in the combination with $\vec{k}$.

Again, for rational $\theta$ and after splitting $\vec{p}$ into its momentum part $\vec{r}$ and its matrix entry part $\vec{\pi}$ the effect of $\vec{r}$ in these terms can be completely undone by an integer shift in the summation variable $\vec{k}$ thus it does not influence the UV behavior (that is $\vec{k}=0$ after Poisson resummation) of the amplitude. Only $\vec{\pi}$, the specification of the matrix entry, has an influence. This is of course expected since the background flux that induces the twisted boundary conditions breaks the degeneracy among the matrix entries. We see that from the point of view if the commutative theory there is no unexpected momentum dependence and thus no UV/IR mixing in the sense explained above.

We find that the regularization provided by the non-commutativity of the coordinates when re-expressed in terms of the commutative theory depends only on the specification of the matrix entry, and not on the commutative momenta. Thus there are no new IR divergencies and therefore no UV/IR mixing.

\section{Spontaneously broken translation invariance and electric flux condensa- tion.}

We have seen that perturbative tachyonic behavior arises at one loop in the pure $U(1)$ gauge theory on a rational non-commutative two-torus. This instability does not occur for the zero modes, which are dual to the free decoupled $U(1)$ modes of the Morita equivalent $U(N)$ theory. The instability of the modes with non-zero momentum suggests they get a non-zero expectation value which spontaneously breaks translation invariance. We shall see that this occurs only for $D=4$, whereas for $D<4$ the apparent symmetry breaking is washed out by quantum fluctuations. In this section we will discuss the phase structure 
of the theory and its interpretation in the Morita equivalent $S U(N) / \mathbb{Z}_{N}$ description ${ }^{3}$. The spontaneous breaking of translation invariance in the non-commutative theory takes a much more familiar form in the Morita equivalent $S U(N) / \mathbb{Z}_{N}$ description, namely that of electric flux condensation. A closely related phenomenon arises in finite temperature deconfinement, which is indicated by the spontaneous breaking of a $\mathbb{Z}_{N}$ symmetry of large gauge transformations on a compactified Euclidean "time" direction [SY1][SY2].

The properties of the map between the non-commutative theory and the $S U(N) / \mathbb{Z}_{N}$ theory which we shall require (see section 2) are the following. For $\theta=p / N$, the gauge coupling of the non-commutative theory $g^{2}$ is equal to the 't Hooft coupling of the commutative theory $g_{\text {com }}^{2} N$. This relation holds when the couplings are evaluated at the same scale. The 't Hooft magnetic flux $m$ is a solution of $p m=1 \bmod N$, and is only defined modulo $N$. The radius of the non-commutative torus $R_{n c}$ is related to that of the commutative torus $R_{\text {com }}$ by $R_{n c}=N R_{\text {com }}$. The momentum on the non-commutative torus is $\vec{n} / R_{n c}$, which is related to the 't Hooft electric flux $\vec{e}$ by $(2.7)$.

This last relation is crucial to us and can be understood in the following way: the 't Hooft electric fluxes of the $S U(N) / \mathbb{Z}_{N}$ theory are measured by operators associated with large gauge transformations on the torus. Upon quotienting by the small gauge transformations, the large gauge transformations generate a $\mathbb{Z}_{N} \times \mathbb{Z}_{N}$ global symmetry. The small gauge transformations satisfy the same boundary conditions as the fields, which are given in $(2.5),(2.6)$, whereas the large gauge transformations satisfy these boundary conditions up to phase factors in the center of $S U(N)$. In the presence of magnetic flux $m$ such that $\operatorname{gcd}(N, m)=1$, the action of the large gauge transformations is equivalent, modulo small gauge transformations, to translating the fields all the way around the commutative torus. Some details concerning large gauge transformations and translations in the torus with twisted boundary conditions are discussed in appendix C. The fact that the translation all the way around the torus is non-trivial is due to the twisted boundary conditions ${ }^{4}$ which give fractional modes in the Fourier expansion of $A_{\mu}$. While one can regard these modes as fractional momenta, a better interpretation is as electric flux. One can then define an integer quantized momentum as

$$
\mathcal{P}_{i}=P_{i}-\epsilon_{i j} e_{j} \frac{m}{N R_{c o m}}
$$

3 Strictly speaking, the Morita dual is a $U(N)$ theory, however we generally ignore the trivial dynamics of the decoupled $U(1)$ sector.

4 Note that the absence of zero modes in the presence of twisted boundary conditions does not by itself break translation invariance, since quantum mechanically, there may still be a translationally invariant vacuum. 
where $\vec{P}$ is the usual generator of translations,

$$
P_{i}=\int_{T^{2} \times \mathbb{R}} d^{3} x \operatorname{tr} F_{i \mu} F_{0 \mu}
$$

The electric fluxes (or fractional momenta) may be reinterpreted as integer momenta upon rewriting the $S U(N) / \mathbb{Z}_{N}$ theory as a non-commutative $U(1)$ gauge theory, due to the relation $R_{n c}=N R_{\text {com }}$. Translations all the way around the commutative torus are equivalent to translations of the non-commutative fields by distances which are an integer multiples of $2 \pi R_{n c} / N$. For this reason condensation of electric flux in the commutative theory via $\mathbb{Z}_{N} \times \mathbb{Z}_{N}$ symmetry breaking is equivalent to spontaneous breaking of translation invariance in the non-commutative description. Of course it may also be viewed as spontaneous breaking of translation invariance in the commutative theory if one takes $\vec{P}$ for the generator of translations rather than $\overrightarrow{\mathcal{P}}$.

Before discussing symmetry breaking, there is a subtlety which we shall address. Since we are considering the case in which two dimensions are compactified on a torus, it might naively appear that the theory is effectively $D-2$ dimensional and that for $D \leq 4$ quantum fluctuations should wash out any symmetry breaking. As is well known, there is no spontaneous breaking of either continuous or discrete global symmetries in theories with dimension less than 2 , and no spontaneous breaking of continuous symmetries [Co] in theories with dimension $2^{5}$. Note that the $\mathbb{Z}_{N} \times \mathbb{Z}_{N}$ symmetry is embedded in a continuous global symmetry generated by $\vec{P}$. However, since the symmetry which is potentially broken is a translation invariance in the compact directions, one must proceed with some care. In the absence of twisted boundary conditions, translation invariance on the torus is not visible after integrating out modes above the scale $1 / R_{\text {com }}$. However with twisted boundary conditions, the fractional momenta survive below the scale $1 / R_{\text {com }}$ and there is a global $\mathbb{Z}_{N} \times \mathbb{Z}_{N}$ symmetry in the dimensionally reduced theory. Furthermore, the continuous symmetry in which this is embedded is no longer visible. To see this explicitly in the $S U(N) / \mathbb{Z}_{N}$ description, it is useful to write the degrees of freedom of the reduced theory in a gauge invariant way. All local gauge invariant operators, such as $\operatorname{trF}^{2}$ are periodic on the commutative torus and have integer momenta. The fractional modes $A^{\mu}(\kappa)$ are associated to the Wilson loops wrapping cycles of the torus (see [vB1],[vB2] and appendix C), which are the creation operators for electric flux. The leading term in the expansion of the path ordered exponential in a Wilson loop is proportional to a fractional momentum mode $A^{\mu}(\kappa)$. Thus below the scale $1 / R_{\text {com }}$ the fundamental degrees of freedom are, up to gauge transformations, $S U(N)$ valued matrices $g_{i}$, from which the wrapped Wilson loops are built (see appendix C). The index $i$ indicates a cycle of the torus. These are the natural

\footnotetext{
5 A possible exception arises in an $N \rightarrow \infty$ limit.
} 
gauge invariant completions of the fractional modes $A_{\mu}(\kappa)$. Note that in the non-compact non-commutative case the one-loop effective action can be written naturally in terms of open Wilson loop operators [KRSY1], [KRSY2], [vR], [AL], [KKRS], which are analogous to the wrapped Wilson loops introduced above.

A general mode with arbitrary fractional momenta and polarization is built from traces of words written with $g_{1}$ and $g_{2}$. The $\mathbb{Z}_{N} \times \mathbb{Z}_{N}$ symmetry acts by multiplying the $g_{i}$ with phases in the center of $S U(N)$. Of course since $g_{i}$ are $S U(N)$ valued, an arbitrary phase is not possible. Moreover there is a cutoff on the fractional momentum arising from the fact that Wilson loops annihilate modulo $N$; traces of sufficiently high powers of $g_{i}$ (i.e. greater than $N$ ) can be written in terms of operators built from traces with smaller powers of $g_{i}$. In this sense, the dimensionally reduced theory below the scale $1 / R_{\text {com }}$ resembles a lattice discretization of the non-commutative torus! Quantum fluctuations in two dimensions present no obstruction to spontaneously breaking the discrete $\mathbb{Z}_{N} \times \mathbb{Z}_{N}$ symmetry.

Let us now examine the phase structure of the four dimensional theory, recalling that the one loop dispersion relation for a photon propagating along a cycle of the two-torus has the general form

$$
E^{2}=\frac{1}{R_{n c}^{2}}\left(\vec{\kappa}^{2}-g^{2} f\left(\frac{\vec{e}}{N}\right)\right)
$$

The gauge coupling in (6.1) should be evaluated at the scale $\kappa_{i} / R$. The function $f\left(\frac{\vec{e}}{N}\right)$ (see (4.10)) depends upon the polarization. As discussed in the previous section, for transverse polarization along the torus this function can lead to instabilities. In these cases, $f$ is positive and takes its largest value when $e_{i}$ is smallest ${ }^{6}$. For small $\vec{e}, f \sim \frac{N^{2}}{\vec{e}^{2}}$.

First let us consider the phase structure as a function of $R$ for fixed $\Lambda_{Q C D}$ and fixed $N$. For $R_{\text {com }} \gg \frac{1}{\Lambda_{Q C D}}$ one expects the energies of electric fluxes to be controlled by a confining linear potential and there should then be no $\mathbb{Z}_{N} \times \mathbb{Z}_{N}$ symmetry breaking via electric flux condensation. At smaller radii, such that the 't Hooft coupling is small, the dispersion relation (6.1) can become tachyonic for sufficiently large $N$, leading to electric flux condensation. However due to asymptotic freedom, the one loop effects of (6.1) become small compared to the kinetic term below a critical radius, leading to symmetry restoration. Thus one has a rather unusual phase diagram, in which symmetry breaking occurs only at intermediate radii. Only the critical point at small radius can be studied perturbatively. The phase transition at this point is apparently second order.

We emphasize that the kinetic term in (6.1) is a feature which arises only in the case of twisted boundary conditions, since only in this case do wrapped Wilson loops

${ }^{6}$ Recall that $f$ vanishes when $e_{i}$ is zero, since such modes correspond to a decoupled sector. 
carry momentum (see appendix C). Thus at tree level, the effect of the twisted boundary conditions is to prevent electric flux condensation. This is very closely related to phenomena arising in twisted Eguchi-Kawai models. Such models have been used to circumvent the $\mathbb{Z}_{N}$ symmetry breaking characteristic of the untwisted Eguchi-Kawai model. However, we find that the one-loop correction can in fact lead to electric flux condensation.

For completeness, let us compare the phase structure of four dimensional $S U(N) / \mathbb{Z}_{N}$ with twisted boundary conditions (magnetic flux) on a $T^{2}$ to that of the same model without magnetic flux. In the latter case, one expects $\mathbb{Z}_{N} \times \mathbb{Z}_{N}$ symmetry breaking at small radius via arguments analogous to those of [SY1], [SY2] in the context of finite temperature deconfinement. The difference in this case is that there is no restoration of the symmetry as the radius goes to zero.

It is interesting to consider the phase structure in various large $N \operatorname{limits}^{7}$. For simplicity we will consider the case in which $\theta=\frac{1}{N}$, or $m=N-1$. The physics of the large $N$ limit depends crucially on how one scales $R_{c o m} \Lambda_{Q C D}$. For instance, keeping $R_{\text {com }} \Lambda_{Q C D}=R_{n c} \Lambda_{Q C D} / N$ and $m / N$ fixed corresponds to a conventional planar 't Hooft limit, whereas scaling $R_{\text {com }} \Lambda_{Q C D}$ like $1 / \sqrt{N}$ describes a decompactification limit of the non-commutative theory $[\mathbf{G}][\mathbf{A B}]$. In the latter case the dimensionful non-commutative parameter $\vartheta=2 \pi \theta R_{n c}^{2}$ is held fixed while $R_{n c} \rightarrow \infty$ like $\sqrt{N}$. However, for a precise discussion the coupling in (6.1) should be evaluated at the proper scale. As the running in the non-commutative theory is non-standard in the infrared [KT] and one must take into account possible finite size complications we will not discuss this issue further here.

When $N$ is large but finite, we expect a non-symmetric but stable vacuum, since it would be very surprising if $S U(N) / \mathbb{Z}_{N}$ Yang-Mills theory on a twisted torus had no stable vacuum. Although it would be interesting to do so, we will not attempt to find the stable vacuum here. Note that the $N \rightarrow \infty$ limit with $R_{\text {com }} \Lambda_{Q C D} \sim 1 / \sqrt{N}$ may be very singular, and it is not clear that the vacuum behaves smoothly in this limit. String theory arguments suggest that the theory on the non-commutative plane has no vacuum [vR], which leads us to presume that there is no such smooth limit.

\section{Conclusions}

We have studied perturbative corrections to correlators for field theories on the noncommutative torus. In the case of the non-commutative plane, these theories are known to show UV/IR-mixing. Loop-corrections that are UV divergent in the commutative limit are regulated by the non-commutativity. On the other hand this regulator is removed for small external momenta and the divergencies reappear.

\footnotetext{
7 Such a study has been performed for a scalar field model in [MRW].
} 
In the compact case an important ingredient is Morita equivalence. It is possible to parameterize the non-commutativity by a dimensionless parameter. This parameterization is not unique but a $S L(2, \mathbb{Z})$ symmetry relates different values. In the case of rational $\theta$ it is possible to reformulate the theory on an ordinary, commutative space. Here, one would not expect an exotic behavior such as UV/IR-mixing.

We have demonstrated that this puzzle can be explained by two observations: first, as the commutative theory is formulated in terms of matrices, what has been momentum in the non-commutative case now has to be split into a label for matrix entries and commutative momentum. Second, as loop integrals are replaced by sums these are subject to Poisson resummations that transform oscillating phase factors into shifts in the sums over momenta. In the rational case, these shifts affect only the matrix entry part and not the commutative momentum part after the Morita map. Thus the commutative theory does not show an unexpected dependence on external momenta.

But even if there is no new dependence on the commutative momenta, the dependence on the matrix entry labels can be quite strong. In fact, for the Moyal bracket interaction and also in the case of the gauge theory, the one loop correction can render some of the modes perturbatively unstable just as it has been known for the theory on the noncommutative plane. We have discussed the nature of these instabilities and their relation to perturbative $\mathbb{Z}_{N} \times \mathbb{Z}_{N}$ symmetry breaking in the gauge theory case.

As the twisted boundary conditions tie large gauge transformations to translations along the cycles of the torus, a breakdown of the symmetry might also be interpreted in terms of spontaneous breaking of translation invariance due to electric flux condensation. It would be very interesting to better understand the nature of this phenomenon and whether it persists in a full non-perturbative quantum formulation of the theory.

Acknowledgments We would like to thank L. Alvarez-Gaumé, J. Barbón, W. Bietenholz, M. García-Pérez, C. Gómez, A. González-Arroyo, C. P. Korthals Altes and D. Lüst for discussions. ZG thanks CERN for hospitality. We also would like to express our gratitude to EG for spaghetti with meatballs. ZG is supported by the DFG under the Emmy Noether programme, grant ER301/1-2, KL is supported by the DFG Schwerpunktprogramm SPP 1096. RCH is supported by the DFG. 


\section{Appendix A: Integral representation of the Epstein Zeta Function}

In this appendix we review the analytic continuation of the Epstein zeta function over the complex $s$-plane using the integral representation in terms of Jacobi-theta functions [M]. For $\operatorname{Re}(s)>1$ the Epstein zeta function is defined through the sum

$$
\zeta(s, \vec{b}):=\sum_{l \in \mathbb{Z}^{2}}^{\prime}\left[(\vec{l}+\vec{b})^{2}\right]^{-s}
$$

We assume that the components of $\vec{b}$ lie both in $\left(-\frac{1}{2}, \frac{1}{2}\right]$. The prime in the sum means that the origin is omitted in the case $\vec{b}=(0,0)$. We can represent this in integral form as

$$
\zeta(s, \vec{b})=\frac{\pi^{s}}{\Gamma(s)} \int_{0}^{\infty} \sum_{l \in \mathbb{Z}_{2}} d t t^{s-1}\left[e^{-\pi t(\vec{l}+\vec{b})^{2}}-\delta_{0, \vec{b}}\right]
$$

In the next step we split the integral from 0 to 1 and from 1 to $\infty$. In the first integral we perform then a Poisson resummation

$$
\sum_{n \in \mathbb{Z}} e^{-a \pi n^{2}+i 2 \pi n b}=\frac{1}{\sqrt{a}} \sum_{l \in \mathbb{Z}} e^{-\frac{\pi}{a}(l+b)^{2}}
$$

and also change the integration variable $t \rightarrow 1 / t$. We integrate the zero-mode in the Poisson resummed expression explicitely and arrive at

$$
\begin{aligned}
\zeta(s, \vec{b})= & \frac{\pi^{s}}{\Gamma(s)}\left(\frac{1}{s-1}-\frac{\delta_{0, \vec{b}}}{s}+\right. \\
& \left.\int_{1}^{\infty} d t\left\{t^{s-1}\left[\sum_{l \in \mathbb{Z}_{2}} e^{-\pi t(\vec{l}+\vec{b})^{2}}-\delta_{0, \vec{b}}\right]+t^{-s}\left[\sum_{n \in \mathbb{Z}_{2}} e^{-\pi t \vec{n}^{2}+i 2 \pi \vec{b} \cdot \vec{n}}-1\right]\right\}\right) .
\end{aligned}
$$

Finally the sums can be written as Products of the Jacobi-Theta function

$$
\vartheta(z, \tau):=\sum_{n \in \mathbb{Z}} e^{i \pi \tau n^{2}+i 2 \pi n z},
$$

which gives the form used in equ. (3.5).

The Epstein zeta function has the following properties. It is meromorphic on the complex $s$-plane with a simple pole at $s=1$ and residue $\pi$. The behavior at the origin is given by $\zeta(0, \vec{b})=-\delta_{0, \vec{b}}$ and more generally $\zeta(-n, \vec{b})=0$ for $n \in \mathbb{N}$. 


\section{Appendix B: Polarization tensor in the NC Torus}

In this Appendix we will derive expression (4.2)-(4.3) for the non-planar part of the polarization tensor. Using the non-commutative Feynman rules, the non-planar contribution to the polarization tensor is given by

$$
\Pi_{\mu \nu}^{N P}=\frac{g^{2}}{(2 \pi R)^{2}} \sum_{\vec{l} \in \mathbb{Z}} \int \frac{d^{d} k}{(2 \pi)^{d}} \frac{\cos \left(2 \pi \theta n_{i} l_{i} \epsilon_{i j}\right)}{\left(k^{2}+\frac{\vec{l}^{2}}{R^{2}}\right)\left((k-p)^{2}+\frac{(\vec{n}-\vec{l})^{2}}{R^{2}}\right)} f_{\mu \nu}\left(K_{\rho}, P_{\sigma}\right),
$$

where $P_{\mu}=\left(p_{\alpha}, n_{i} / R\right)$ is the inflowing momentum, $K_{\mu}=\left(k_{\alpha}, l_{i} / R\right)$ denotes the loop momentum and $f_{\mu \nu}\left(L_{\rho}, P_{\sigma}\right)$ is

$4 d K_{\mu} K_{\nu}-2 d\left(P_{\mu} K_{\nu}+P_{\nu} K_{\mu}\right)+(d-4) P_{\mu} P_{\nu}-g_{\mu \nu}\left(2 d K^{2}-(4 d+2) K \cdot P+(2 d-3) P^{2}\right)$.

In order to evaluate the previous expression we introduce Feynman and Schwinger parameters, which allow us to easily integrate the internal momentum along the noncompact directions, $k_{\alpha}$. We expand then the cosine in terms of exponentials and Poisson resum, with the result

$$
\Pi_{\mu \nu}^{N P}=\frac{g^{2}}{2(4 \pi)^{\frac{d}{2}+1}} \sum_{\epsilon= \pm 1} \sum_{\vec{l} \in \mathbb{Z}^{2}} \int_{0}^{\infty} \frac{d \alpha}{\alpha^{\frac{d}{2}}} \int_{0}^{1} d x e^{-2 \pi i \epsilon x P \cdot L} e^{-\alpha P^{2} x(1-x)-\frac{\pi^{2}}{\alpha} L^{2}} f_{\mu \nu}\left(P_{\rho}, L_{\sigma}\right),
$$

where we have set for simplicity $R=1$. We recall that $L_{\mu}=\left(0, l_{i}-\theta \epsilon_{i j} n_{j}\right)$. The polynomial $f_{\mu \nu}$ after these manipulations reads

$$
\begin{aligned}
f^{\prime}{ }_{\mu \nu}\left(P_{\rho},\right. & \left.L_{\sigma}\right)=-\frac{2 d \pi^{2}}{\alpha^{2}}\left[2 L_{\mu} L_{\nu}-g_{\mu \nu}\left(L^{2}-\frac{d}{2 \pi^{2}} \alpha\right)\right]- \\
- & \frac{i \pi \epsilon}{\alpha}\left[2 d(2 x-1)\left(P_{\mu} L_{\nu}+P_{\nu} L_{\mu}\right)+g_{\mu \nu}(4 d(1-x)+2) P \cdot L\right]+ \\
+ & (4 d x(1-x)-d+4) P_{\mu} P_{\nu}-g_{\mu \nu}\left(2 d(x-1)^{2}-2 x-3\right) P^{2} .
\end{aligned}
$$

This complicated expression can be simplified by making use of the properties of Bessel functions. Let us start by concentrating our attention on the first line in the rhs of (9.2). Since it carries negative powers of the Schwinger parameter $\alpha$, it can provide very large contributions from the UV region $\alpha \rightarrow 0$. Notice that the second term inside the square bracket is proportional to $g_{\mu \nu}$ and thus could threaten the Ward identities. However a cancellation takes place, as can be seen from the following relations

$$
\begin{aligned}
\int_{0}^{\infty} \frac{d \alpha}{\alpha^{\frac{d}{2}+2}} e^{-z^{2} \alpha-\frac{1}{\alpha}}\left(1-\frac{d}{2} \alpha\right) & =2 z^{\frac{d}{2}+1}\left(K_{\frac{d}{2}+1}(2 z)-\frac{d}{2 z} K_{\frac{d}{2}}(2 z)\right)= \\
& =2 z^{\frac{d}{2}+1} K_{\frac{d}{2}-1}(2 z)=z^{2} \int_{0}^{\infty} \frac{d \alpha}{\alpha^{\frac{d}{2}}} e^{-z^{2} \alpha-\frac{1}{\alpha}} .
\end{aligned}
$$


This relation applies to (9.1)-(9.2) by setting $z^{2}=\pi^{2} L^{2} P^{2} x(1-x)$. It implies that we can substitute

$$
-\frac{2 d}{\alpha^{2}} g_{\mu \nu}\left(\pi^{2} L^{2}-\frac{d}{2} \alpha\right) \rightarrow-2 d P^{2} x(1-x)
$$

in ${f^{\prime}}_{\mu \nu}$, allowing us to rewrite it in the form

$$
\begin{aligned}
f_{\mu \nu}^{\prime \prime}\left(P_{\rho}, L_{\sigma}\right)= & -\frac{4 d \pi^{2}}{\alpha^{2}} L_{\mu} L_{\nu}-\frac{2 \pi i d \epsilon}{\alpha}(2 x-1)\left[P_{\mu} L_{\nu}+P_{\nu} L_{\mu}-g_{\mu \nu} P \cdot L\right] \\
& -\left(P_{\mu} P_{\nu}-g_{\mu \nu} P^{2}\right)(4 d x(1-x)-d+4)+ \\
& +g_{\mu \nu}(d+1)\left[(2 x-1) P^{2}-\frac{2 i \epsilon \pi}{\alpha} P \cdot L\right] .
\end{aligned}
$$

In order to proceed we will show that the following equation holds for $r \in \mathbb{Z}$

$$
\begin{aligned}
\int_{0}^{1} d x e^{2 \pi i r x} & (2 x-1) \int_{0}^{\infty} \frac{d \alpha}{\alpha^{n}} e^{-\alpha z^{2} x(1-x)-\frac{1}{\alpha}}= \\
= & -\frac{2 \pi i r}{z^{2}} \int_{0}^{1} d x e^{2 \pi i r x} \int_{0}^{\infty} \frac{d \alpha}{\alpha^{n+1}} e^{-\alpha z^{2} x(1-x)-\frac{1}{\alpha}}
\end{aligned}
$$

The lhs side of this equation is

$$
\begin{aligned}
\int_{0}^{1} d x & e^{2 \pi i r x}(2 x-1) \int_{0}^{\infty} \frac{d \alpha}{\alpha^{n}} e^{-\alpha z^{2} x(1-x)-\frac{1}{\alpha}}= \\
& =2 \int_{0}^{1} d x e^{2 \pi i r x}(2 x-1)(z \sqrt{x(1-x)})^{n-1} K_{n-1}(2 z \sqrt{x(1-x)})= \\
& =\frac{2}{z^{2}} \int_{0}^{1} d x e^{2 \pi i r x} \partial_{x}\left[(z \sqrt{x(1-x)})^{n} K_{n}(2 z \sqrt{x(1-x)})\right]=
\end{aligned}
$$

where we have used that $\partial_{y}\left(y^{n} K_{n}(y)\right)=-y^{n} K_{n-1}(y)$. Provided $r$ is an integer, integrating by parts we obtain the desired relation

$$
\begin{aligned}
& =-\frac{4 \pi i r}{z^{2}} \int_{0}^{1} d x e^{2 \pi i r x}(z \sqrt{x(1-x)})^{n} K_{n}(2 z \sqrt{x(1-x)})= \\
& =-\frac{2 \pi i r}{z^{2}} \int_{0}^{1} d x e^{2 \pi i r x} \int_{0}^{\infty} \frac{d \alpha}{\alpha^{n+1}} e^{-\alpha z^{2} x(1-x)-\frac{1}{\alpha}} .
\end{aligned}
$$

Setting $r=\epsilon P \cdot L$ and $z^{2}=\pi^{2} L^{2} P^{2}$, the equality (9.4) implies that we can replace

$$
(2 x-1) \rightarrow \frac{2 i \epsilon \pi}{\alpha} \frac{P \cdot L}{P^{2}}
$$

in the involved expression of ${f^{\prime \prime}}_{\mu \nu}$. It is clear then that the last line in (9.3) cancels, and $f^{\prime \prime}{ }_{\mu \nu}$ reduces to

$$
\begin{aligned}
h_{\mu \nu}\left(P_{\rho}, L_{\sigma}\right)= & -\frac{4 d \pi^{2}}{\alpha^{2}}\left[L_{\mu} L_{\nu}-\left(P_{\mu} L_{\nu}+P_{\nu} L_{\mu}-g_{\mu \nu} P \cdot L\right) \frac{P \cdot L}{P^{2}}\right]- \\
& -\left(P_{\mu} P_{\nu}-g_{\mu \nu} P^{2}\right)(4 d x(1-x)-d+4) .
\end{aligned}
$$




\section{Appendix C: Translations and large gauge transformations on a twisted torus}

In this appendix we review some general properties of translations and large gauge transformations on a twisted two-torus. We consider an $S U(N) / \mathbb{Z}_{N}$ gauge theory on a torus with twisted boundary conditions corresponding to magnetic flux $m$. The $S U(N)$ valued twist matrices associated to the cycles of the torus are $U_{1}$ and $U_{2}$ which satisfy

$$
U_{1} U_{2}=U_{2} U_{1} \exp \left(-2 \pi i \frac{m}{N}\right),
$$

and may be chosen to be constant. Translating the gauge fields all the way around the cycles of the torus is equivalent to acting with the gauge transformations $U_{1}$ and $U_{2}$.

Physical states are invariant under Small gauge transformations. These are continously connected to the identity and are described by $U_{s}(x)$ satisfying

$$
U_{s}\left(x^{i}+2 \pi R^{i}\right)=U_{i} U_{s}(x) U_{i}^{\dagger} .
$$

However, there are more transformations that preserve the boundary conditions for the connection. In addition to the small transformations there are transformations for which $U_{s}(x)$ obeys (10.1) only up to a phase. This phase has to be an $N$ th root of unity in order for $U_{s}$ to be in $S U(N)$ :

$$
U_{l}\left(x^{i}+2 \pi R^{i}\right)=U_{i} U_{l}(x) U_{i}^{\dagger} \exp \left(2 \pi i \frac{n_{i}}{N}\right)
$$

Note that the twists $U_{1}$ and $U_{2}$ are of this type. For instance

$$
U_{2}=U_{1} U_{2} U_{1}^{\dagger} \exp (2 \pi i m / N)
$$

There is a $\mathbb{Z}_{N} \times \mathbb{Z}_{N}$ global symmetry associated to the quotient of large gauge transformations by small ones. For the case $\operatorname{gcd}(N, m)=1$, which is dual a non-commutative $U(1)$ theory, this symmetry is completely generated by the twist matrices, whereas in other cases the twist matrices only generate a subgroup.

States that transform non-trivially under large gauge transformations are the 't Hooft electric fluxes. The associated creation operators are wrapped Wilson loops. Because of the relation between the large gauge transformations and the twists, or translations all the way around the torus, one expects such operators to have momentum which is fractional in units of $1 / \mathrm{N}$. For instance the Wilson loops which wrap the $i$ th cycle of the torus $e$ times are given by

$$
W_{i, e}=\operatorname{tr} P\left(e^{i \oint_{e S_{i}} A_{i}}\right) U_{i}^{\dagger^{e}}
$$


where the twist matrix in this expression is necessary for the operator to be invariant under small gauge transformations [vB1], [vB2]. Note that this can also be written as

$$
W_{i, e}=\operatorname{tr} g_{i}^{e}
$$

where

$$
g_{i}=P\left(e^{i \oint_{S_{i}} A_{i}}\right) U_{i}^{\dagger}
$$

Upon translating all the way around the torus in the transverse direction, this operator picks up the phase $\exp (2 \pi i m e / N)$. In an effective action for the fractional momentum modes, these Wilson loop operators are the natural gauge invariant completions of gauge field modes with fractional momenta. For example, expanding the exponential gives

$$
\operatorname{tr} g_{1}^{e_{1}}=2 \pi \kappa_{2} R_{c o m} A_{1}\left(\kappa_{2}\right)+\ldots
$$

In a non-abelian gauge theory, the translation generator $p_{j}=\int \operatorname{tr} F_{0 i} \partial_{j} A_{i}$ is not gauge invariant. However the gauge invariant version $P_{j}=\int \operatorname{tr} F_{0 i} F_{j i}$ generates exactly the same action on physical states, since the action of the two generators differs by a small gauge transformation. For instance, the infinitesimal translation $\exp \left(i \epsilon p_{j}\right)$ differs from $\exp \left(i \epsilon P_{j}\right)$ by a gauge transformation with the gauge parameter $\alpha=\epsilon A_{j}$. A translation all the way around the torus is generated by $\exp \left(2 \pi i R P_{j}\right)$, which in light of our previous discussion is a large gauge transformation. Thus when there is a non-zero electric flux, the momentum $P_{j}$ may be fractional in units of $1 / N$. The fractional momenta are better interpreted as electric fluxes. One can define a momentum with integer eigenvalues by

$$
\mathcal{P}_{j} \equiv P_{j}-m \epsilon_{j i} e_{i} / N
$$

\section{References}

[AB] Alvarez-Gaume, L. and Barbon, J. L. F.: "Morita duality and large-N limits", Nucl. Phys. B623 (2002) p. 165-200 hep-th/0109176

[AL] Armoni, Adi and Lopez, Esperanza: "UV/IR mixing via closed strings and tachyonic instabilities", hep-th/0110113

[BGNV] Bassetto, A. and Griguolo, L. and Nardelli, G. and Vian, F. : "On the unitarity of quantum gauge theories on noncommutative spaces", JHEP 07 (2001) p. 008 hep-th/0105257 
[C] Connes, Alain: "Noncommutative Geometry" (1994) Academic Press

[CMS] Chu, Chong-Sun and Madore, John and Steinacker, Harold: "Scaling limits of the fuzzy sphere at one loop", JHEP 08 (2001) p. 038 hep-th/0106205

[Co] Coleman, Sidney R.: "There are no Goldstone bosons in two-dimensions", Commun. Math. Phys. 31 (1973) p. 259-264

[DFR] Doplicher, S. and Fredenhagen, K. and Roberts, J. E.: "The Quantum structure of space-time at the Planck scale and quantum fields", Commun. Math. Phys. 172 (1995) p. 187-220

[DN] Douglas, Michael R. and Nekrasov, Nikita A.: "Noncommutative field theory", Rev. Mod. Phys. 73 (2002) p. 977-1029 hep-th/0106048

[F] Filk, T.: "Divergencies in a field theory on quantum space", Phys. Lett. B376 (1996) p. 53-58

[G] Guralnik, Zachary: "Strong coupling phenomena on the noncommutative plane", hep-th/0109079

[GK] Gonzalez-Arroyo, A. and Korthals Altes, C. P.: "Reduced Model For Large N Continuum Field Theories", Phys. Lett. B131 (1983) p. 396

[GMW] Gomis, Jaume and Mehen, Thomas and Wise, Mark B.: "Quantum field theories with compact noncommutative extra dimensions", JHEP 08 (2000) p. 029 hep-th/0006160

[GP] Griguolo, Luca and Pietroni, Massimo: "Wilsonian renormalization group and the non-commutative IR/UV connection", JHEP 05 (2001) p. 032 hepth/0104217

[GT] Zachary Guralnik and Jan Troost: "Aspects of gauge theory on commutative and noncommutative tori", hep-th/0103168

[H] Helling, Robert C.: "A remark on field theories on the non-commutative torus", hep-th/0111077

[Ha] Hayakawa, M.: "Perturbative ultraviolet and infrared dynamics of noncommutative quantum field theory", hep-th/0009098 
[KKRS] Kiem, Young-jai and Kim, Yeon-jung and Ryou, Cheol and Sato, Haru-Tada: "One-loop noncommutative U(1) gauge theory from bosonic worldline approach", hep-th/0112176

[KRSY1] Kiem, Youngjai and Rey, Soo-Jong and Sato, Haru-Tada and Yee, Jung-Tay: "Open Wilson lines and generalized star product in nocommutative scalar field theories", Phys. Rev. D65 (2002) p. 026002 hep-th/0106121

[KRSY2] Kiem, Youngjai and Rey, Soo-Jong and Sato, Haru-Tada and Yee, JungTay: "Anatomy of one-loop effective action in noncommutative scalar field theories", Eur. Phys. J. C22 (2002) p. 757-770 hep-th/0107106

[KT] Khoze, Valentin V. and Travaglini, Gabriele: "Wilsonian effective actions and the IR/UV mixing in noncommutative gauge theories", JHEP 01 (2001) p. 026 hep-th/0011218

[KW] Krajewski, Thomas and Wulkenhaar, Raimar: "Perturbative quantum gauge fields on the noncommutative torus", Int. J. Mod. Phys. A15 (2000) p. 1011-1030 hep-th/9903187

[LLT1] Landsteiner, Karl and Lopez, Esperanza and Tytgat, Michel H. G.: "Excitations in hot non-commutative theories", JHEP 09 (2000) p. 027 hepth/0006210

[LLT2] Landsteiner, Karl and Lopez, Esperanza and Tytgat, Michel H. G.: "Instability of non-commutative SYM theories at finite temperature", JHEP 06 (2001) p. 055 hep-th/0104133

[M] David Mumford: "Tata Lectures on Theta I, (chap. I.16)" (1983) Birkhäuser Boston-Basel-Stuttgart

[MRW] Mandal, Gautam and Rey, Soo-Jong and Wadia, Spenta R.: "Quantum aspects of GMS solutions of noncommutative field theory and large $\mathrm{N}$ limit of matrix models", hep-th/0111059

[MST] Matusis, Alec and Susskind, Leonard and Toumbas, Nicolaos: "The IR/UV connection in the non-commutative gauge theories", JHEP 12 (2000) p. 002 hep-th/0002075 
[MvRS] Minwalla, Shiraz and Van Raamsdonk, Mark and Seiberg, Nathan: "Noncommutative perturbative dynamics", JHEP 02 (2000) p. 020 hep-th/9912072

[PS] Peskin, Michael E. and Schroeder, D. V.: "An Introduction to quantum field theory", Reading, USA: Addison-Wesley (1995) $842 \mathrm{p}$

[RR] Ruiz, F. Ruiz: "Gauge-fixing independence of IR divergences in non- commutative U(1), perturbative tachyonic instabilities and supersymmetry", Phys. Lett. B502 (2001) p. 274-278 hep-th/0012171

[S] Saraikin, Kirill: "Comments on the Morita equivalence", J. Exp. Theor. Phys. 91 (2000) p. 653-657 hep-th/0005138

[Sn] Snyder, Hartland S.: "Quantized space-time", Phys. Rev. 71 (1947) p. 3841

[SY1] Svetitsky, Benjamin and Yaffe, Laurence G.: "Critical behaviour at finite temperature confinement transitions", Nucl. Phys. B210 (1982) p. 423

[SY2] Yaffe, L. G. and Svetitsky, B.: "First order phase transitions in the $S U(3)$ gauge theory at finite temperature", Phys. Rev. D26 (1982) p. 963

[SZ] Schwarz, Albert: "Morita equivalence and duality", Nucl. Phys. B534 (1998) p. $720-738$ hep-th/9805034

[vB1] van Baal, Pierre: "QCD in a finite volume", hep-ph/0008206

[vB2] van Baal, Petrus Jacobus: "Twisted boundary conditions: A nonperturbative probe for pure nonabelian gauge theories", PhD thesis, INIS-mf-9631

[vR] Van Raamsdonk, Mark: "The meaning of infrared singularities in noncommutative gauge theories", JHEP 11 (2001) p. 006 hep-th/0110093 